\title{
Comparison of inhaled salmeterol and individually dose- titrated slow-release theophylline in patients with reversible airway obstruction
}

\author{
P.L. Paggiaro*, D. Giannini*, A. Di Franco*, R. Testi** \\ on behalf of a European Study Group
}

Comparison of inhaled salmeterol and individually dose-titrated slow-release theophylline in patients with reversible airway obstruction. P.L. Paggiaro, D. Giannini, A. Di Franco, R. Testi on behalf of a European Study Group. CERS Journals Ltd 1996.

ABSTRACT: The aim of this study was to compare the clinical efficacy of salmeterol versus theophylline in the treatment of moderate-to-severe asthma.

One hundred and eighty nine asthmatic patients (forced expiratory volume in one second (FEV1) or peak expiratory flow (PEF) $>50 \%$ of predicted) were randomized to receive either salmeterol dry powder, $50 \mu \mathrm{g}$ b.i.d. via a Diskhaler $(\mathrm{n}=92)$ or dose-titrated slow-release theophylline capsules ("Theo-Dur") b.i.d. (n=97), in a double-blind, double-dummy, parallel group study for 4 weeks. Patients had previously been titrated with theophylline to a serum theophylline level of $10-20 \mu \mathrm{g} \cdot \mathrm{mL}^{-1}$.

The median percentage of nights with no asthma symptoms rose from $14 \%$ in both groups at baseline to $71 \%$ with salmeterol and to $46 \%$ with theophylline $(p=0.044)$. There was also a significant increase for salmeterol in the median percentage of nights with no rescue salbutamol use (from 36 to $86 \%$ ) compared with theophylline (from 71 to $78 \%$; $\mathrm{p}=\mathbf{0 . 0 0 2}$ ). The mean morning PEF increased from $337 \mathrm{~L} \cdot \mathrm{min}^{-1}$ in the salmeterol group and $332 \mathrm{~L} \cdot \mathrm{min}^{-1}$ in the theophylline group to 372 and $357 \mathrm{~L} \cdot \mathrm{min}^{-1}$, respectively. No significant difference between the two treatments was observed for PEF, symptoms or additional salbutamol medication during the day. The incidence of gastrointestinal symptoms (gastric irritation, nausea and vomiting) was greater among patients receiving theophylline $(11 \%)$ than with salmeterol $(3 \%)$.

These findings suggest that inhaled salmeterol is more effective in relieving symptoms of asthma, and better tolerated than theophylline in patients with moderateto-severe asthma.

Eur Respir J., 1996, 9, 1689-1695. *2nd Institute of Internal Medicine, Respiratory Pathophysiology, University of Pisa, Italy. **Glaxo Company, Verona, Italy.

Correspondence: P.L. Paggiaro

Fisiopatologia Respiratoria

Ospedale Cisanello

via Paradisa 2

56100 Pisa

Italy

Keywords: Asthma treatment

$\beta$-agonists

bronchodilator drugs

salmeterol

theophylline

Received: February 131995

Accepted after revision April 241996

This study was supported by Glaxo Group Research, UK.
The clinical efficacy of salmeterol, the first of a new class of long-acting $\beta_{2}$-agonists, has been demonstrated in comparison with other short-acting $\beta_{2}$-agonists, such as salbutamol and terbutaline [1,2]. As oral theophylline is an asthma drug widely used in many countries, especially in Europe, this study was planned to compare the effects of salmeterol with oral slow-release theophylline. The new slow-release preparations of oral theophylline allow quite stable levels of serum theophylline concentration with one or two daily administrations, and they are commonly accepted for treatment of chronic reversible airway obstruction [3]. In this respect, the comparison between oral slow-release theophylline and salmeterol, both drugs with a long-lasting (up to $12 \mathrm{~h}$ ) bronchodilation achieved by twice daily administration, seems of particular interest for long-term treatment of reversible airway obstruction. In a previous study, the effect of salmeterol was compared with theophylline over 2 weeks in a double-blind, cross-over study [4], showing that salmeterol produced a better effect on asthma symptoms and peak expiratory flow (PEF) values than theophylline in subjects with moderate asthma. Salmeterol was also shown to be more effective than a combination of slow-release theophylline and ketotifen in producing disappearance of nocturnal symptoms, lung function and rescue salbutamol use, during a 2 week period of treatment in 115 patients with nocturnal asthma [5].

This large multicentre study was conducted in 24 European Centres, in order to compare the efficacy and tolerability of salmeterol with oral theophylline in the treatment of moderate-to-severe asthma.

\section{Subjects and methods}

\section{Subjects}

Two hundred and forty three patients with moderateto-severe asthma were recruited into the study. Inclusion criteria were: 1) male or female aged 18-70 yrs; 2) symptoms of asthma (dyspnoea, wheeze, cough) for at least 1 year; 3) reversible airway obstruction (forced expiratory volume in one second (FEV1) increase of more than $15 \%$ from the baseline value after $400 \mu \mathrm{g}$ dry powder inhaled salbutamol by Diskhaler) in one of two visits 
during the run-in period; and 4) airway obstruction (FEV1 or mean $\mathrm{PEF}>50 \%$ of the predicted value). Patients with respiratory tract infections or hospitalizations due to an exacerbation of asthma within 30 days prior to the study were not eligible. Patients had to be able to give informed consent to the study, and to regularly record daytime and night-time symptoms and PEF measurements twice daily. Regular use of inhaled anti-inflammatory drugs was allowed, and had to remain constant throughout the study period.

Exclusion criteria were: the presence of other serious cardiopulmonary and disabling diseases; intolerance to inhaled $\beta_{2}$-agonists or oral theophylline (e.g. adverse effects during previous experience with these drugs); pregnancy; and severe steroid-dependent asthma.

The study was approved by the Ethics Committees of the different centres which participated.

\section{Study design}

The study consisted of two 1 week run-in periods, during which the patients continued their regular treatment for asthma, with the exception of oral theophylline and regular oral or inhaled $\beta_{2}$-agonists. Daytime and nighttime symptoms score, use of additional salbutamol medication, and morning and evening PEF were measured each day to obtain the baseline evaluation. Patients were eligible for the study if they fulfilled inclusion criteria and demonstrated during the run-in period a score of asthma symptoms during night and day of two or more (with a score of one or more in the daytime) or a diurnal variation of PEF $>15 \%$ in 4 out of 7 days during the last week. Titration of theophylline was then performed in order to obtain, in each patient, a peak serum theophylline level of $10-20 \mu \mathrm{g} \cdot \mathrm{mL}^{-1}$; each eligible patient received $150 \mathrm{mg}$ b.i.d. of slow-release oral theophylline (Theo-Dur), and the dose was increased by $150 \mathrm{mg}$ b.i.d. at intervals of 4-7 days until a serum theophylline level of $10-20 \mu \mathrm{g} \cdot \mathrm{mL}^{-1}$ was achieved. Patients who did not reach a theophylline level $>10 \mu \mathrm{g} \cdot \mathrm{mL}^{-1}$ after a dose of $600 \mathrm{mg}$ b.i.d. were withdrawn.

Patients were then randomized into two treatment groups. The trial consisted of a 4 week, randomized, double-blind, double-dummy, placebo-controlled, parallel group study. The active treatments to be compared were: 1) salmeterol $50 \mu \mathrm{g}$ b.i.d. by dry-powder inhalation; and 2) oral slow-release theophylline b.i.d. at doses previously titrated in each subject to obtain a serum theophylline concentration of $10-20 \mu \mathrm{g} \cdot \mathrm{mL}^{-1}$. Other concomitant treatment was allowed, except for theophylline, anticholinergics or regular $\beta_{2}$-agonists, and they were maintained at the same dose throughout the study period. Regular anti-inflammatory treatment with inhaled corticosteroids, cromoglycate, nedocromil or systemic steroids was used by 103 out of 189 patients ( 52 in the salmeterol group and 51 in theophylline group), and the doses remained constant during the study. At the end of the active treatment patients underwent a 2 week follow-up period, during which concurrent medications only were allowed.

\section{Criteria of response}

Diary card. Throughout the study, the patient recorded the severity of his own asthma symptoms, every morning and every evening, by means of a symptom score: from 0 (no symptoms at all) to 5 (severe symptoms, which made it impossible to attend work) for daytime symptoms; and from 0 to 4 (severe symptoms, which made it impossible to sleep all night) for night-time symptoms. The number of salbutamol inhalations for the relief of asthmatic symptoms during the night and the day was also recorded.

Peak expiratory flow. The patients were instructed to measure PEF every day in the early morning and in the evening, using a mini-Wright peak flow meter, before the administration of the study drug. They performed three acceptable manoeuvres, and the highest value was recorded on the diary card. They also had to record the use of additional salbutamol in the $4 \mathrm{~h}$ prior to PEF measurements.

Serum theophylline measurement. Serum theophylline was measured during the titration period, on a sample of whole blood taken by puncture from the finger, using a simple and accurate enzyme immunochromatography technique (Acculevel Theophylline Assay Kit), which made it possible to obtain the results in less than $15 \mathrm{~min}$. The measurement was performed in the laboratory, 3-4 $\mathrm{h}$ after the last administration of theophylline or placebo capsules. At the same time, a further sample of serum taken by venous puncture was stored for subsequent analysis at a central laboratory.

Clinical evaluation. At each visit to the laboratory, in the late morning, the patients measured FEV1, forced vital capacity (FVC) and other indices derived from the flow-volume curve, 3-4 $\mathrm{h}$ after having received the morning dose of the study drugs as performed on each day of the study. The spirometric equipment of the different centres was periodically checked (every month at least) by $3 \mathrm{~L}$ volume syringe calibration; accuracy should be maintained between $\pm 1 \%$, as reported by the American Thoracic Society (ATS) recommendations. The values were expressed in percentage of the predicted values [6]. Vital signs, i.e. systolic and diastolic blood pressure, heart rate and weight were recorded at each visit. Haematological evaluation was performed at the beginning of the study (to exclude important concomitant diseases) and at the end of the active treatment (to detect for any subclinical adverse event); it was eventually repeated during the follow-up after the end of the study. An independent assessment by the physician and by the patient on the efficacy of the treatment was made during and at the end of the active treatment. Compliance to the treatment was evaluated by the investigator; pills or Diskhalers used by the patient were collected by the investigator at each visit, but they were not counted.

Adverse events. Patients were asked about major and minor adverse events, which were recorded irrespective of their apparent cause in relationship to the study drugs. The physician checked the daily diary card and enquired directly whether the patient had any other problems since the previous clinic visit. 


\section{Statistical analysis}

After validation, all record forms were included in the data base and checked for accuracy and consistency. All statistical analyses were performed using either Statistical Analysis System (SAS) (Release 6.06) or RS1 under VMS (Digital Equipment Corporation) operating system. Symptom and drug score (percentage of days or nights with asthma score of zero, median daytime or night-time asthma score) and mean PEF measurements were evaluated during 4 weeks of active treatment, in comparison with the 2 nd week of the run-in period (baseline evaluation) for each treatment. Analysis of covariance (ANCOVA) was carried out on PEF measurements, using the corresponding derived baseline variables as covariates, with adjustment for variation due to country, baseline response and treatment; the mean difference between treatments was estimated with $95 \%$ confidence limits (95\% CL), and examined for significance using a t-test.

For each patient, the increase between the baseline and the treatment period in the percentage of days or nights with a symptom score of zero, or with no additional salbutamol, was calculated. In order to assess the significance of treatment differences, Wilcoxon rank sum analysis was performed on the increase in the percentage of days. This analysis was also performed with the data stratified by country (Van Elteren test). Data from clinic evaluation were tested by ANCOVA, adjusting for country, baseline value and treatment; the mean difference between treatments was estimated (with 95\% CL) and tested for significance by a t-test. Adverse events were summarized and tabulated and compared using a Fisher exact test.

\section{Results}

Fifty four patients were withdrawn prior to randomization, mainly because they did not meet the eligibility criteria. One hundred and eighty nine patients entered the study: 92 were treated with dry power inhaled salmeterol (50 $\mathrm{\mu g}$ b.i.d. by Diskhaler) and 97 with oral theophylline.

No significant difference could be observed at the first examination between patients treated with salmeterol and patients treated with oral theophylline as regards demography (male/female ratio, age, FEV1, smoking habit, duration of asthma, prevalence of atopy) (table 1) and history of reversible airway obstruction (number of exacerbations in the last year, causes of exacerbations). More than $50 \%$ of subjects in both groups were lifelong nonsmokers; mean pack-years of smokers were 10.5 \pm 16.2 and $20.6 \pm 16.8$ in the salmeterol $(n=14)$ and theophylline $(n=18)$ group, respectively.

Although inclusion criteria required a FEV1 value $>50 \%$ of the predicted value, 14 out of $189(8 \%)$ patients (seven in the salmeterol group and seven in the theophylline group had a FEV $1<50 \%$ predicted). All patients had a reversibility test in the run-in period; in all but eight patients the increase in FEV1 after salbutamol, in percentage of the baseline value, was $>15 \%$, with a simultaneous increase in FEV 1 of at least $200 \mathrm{~mL}$. When reversibility was expressed as increase in FEV1 after salbutamol as a percentage of the predicted value, only
Table 1. - Demography of the asthmatic patients who received active treatment

\begin{tabular}{|c|c|c|}
\hline & Salmeterol & Theophylline \\
\hline Patients $\mathrm{n}$ & 92 & 97 \\
\hline $\operatorname{Sex} \quad M / F$ & $54 / 38$ & $52 / 45$ \\
\hline Age $\#$ yrs & $42(18-77)$ & $43(17-78)$ \\
\hline FEV $1 \%$ pred & $68(38-143)$ & $72(26-150)$ \\
\hline \multicolumn{3}{|l|}{ Smoking habit* $\mathrm{n}$} \\
\hline Current smoker & $14(15)$ & $18(18)$ \\
\hline Nonsmoker & $51(55)$ & $59(61)$ \\
\hline Ex-smoker & 27 (29) & $20(21)$ \\
\hline \multicolumn{3}{|l|}{ Duration of asthma* $n$} \\
\hline $0-10$ yrs & $51(56)$ & $61(63)$ \\
\hline $11-20 \mathrm{yrs}$ & $25(27)$ & $22(23)$ \\
\hline $21-30 \mathrm{yrs}$ & 7 (8) & 9 (9) \\
\hline$>30 \mathrm{yrs}$ & $8 \quad(9)$ & $5 \quad(5)$ \\
\hline Not recorded & 1 & \\
\hline \multicolumn{3}{|l|}{ Evidence of atopy* $n$} \\
\hline Positive skin tests & $40(48)$ & 39 (49) \\
\hline Raised serum IgE & $32(52)$ & $33(50)$ \\
\hline Eosinophilia & $25(36)$ & $32(40)$ \\
\hline Seasonal rhinitis & $30(33)$ & $31(32)$ \\
\hline Eczema & $15(16)$ & $14(14)$ \\
\hline Family asthma history & $43(47)$ & 38 (39) \\
\hline \multicolumn{3}{|c|}{ Prestudy asthma medication* $n$} \\
\hline Beta-agonists & $63(68)$ & $70(72)$ \\
\hline Inhaled steroids & 43 (47) & $46(47)$ \\
\hline Systemic steroids & 11 (12) & 7 (7) \\
\hline Methylxanthines & 31 (34) & $35(36)$ \\
\hline DSCG or nedocromil & $6 \quad(7)$ & $5 \quad(5)$ \\
\hline Anticholinergics & $15(16)$ & $9 \quad(9)$ \\
\hline Ketotifen & 6 (7) & $3 \quad(3)$ \\
\hline Other medications & $5 \quad(5)$ & $5 \quad(5)$ \\
\hline
\end{tabular}

\#: median, and range in parenthesis; *: absolute value, and percentage in parenthesis. M: male; F: female; FEV1: forced expiratory volume in one second; IgE: immunoglobulin E; DSCG: disodium cromoglycate; $\%$ pred: percentage of predicted value.

15 patients $(8 \%)$ showed a $<9 \%$ increase (seven in the salmeterol group and eight in the theophylline group).

Most patients in both groups were receiving regular treatment with inhaled or systemic steroids, or cromoglycate; 66 and $64 \%$ of patients treated with salmeterol or theophylline, respectively, during active treatment, had not been treated previously with theophylline (table 1).

Concurrent medications taken during the active treatment period are reported in table 2. The percentage of treatments with inhaled or systemic anti-inflammatory drugs was high and similar in both treatment groups.

Daytime and night-time symptom scores were reduced during the 4 weeks of active treatment in both groups,

Table 2. - Concurrent asthma medications taken by the asthmatic patients during the active treatment period

\begin{tabular}{lrrrr}
\hline & \multicolumn{3}{c}{$\begin{array}{c}\text { Salmeterol } \\
\mathrm{n}\end{array}$} & $\begin{array}{r}\text { Theophylline } \\
\mathrm{n}\end{array}$ \\
& & & & $(\%)$ \\
\hline Steroids & & & & \\
$\quad$ Inhaled only & 40 & $(43)$ & 41 & $(42)$ \\
$\quad$ Systemic & 8 & $(9)$ & 5 & $(5)$ \\
DSCG or nedocromil & 4 & $(4)$ & 5 & $(5)$ \\
Ketotifen & 4 & $(4)$ & 1 & $(1)$ \\
Other medications & 4 & $(4)$ & 3 & $(3)$ \\
& & & &
\end{tabular}

DSCG: disodium cromoglycate. 

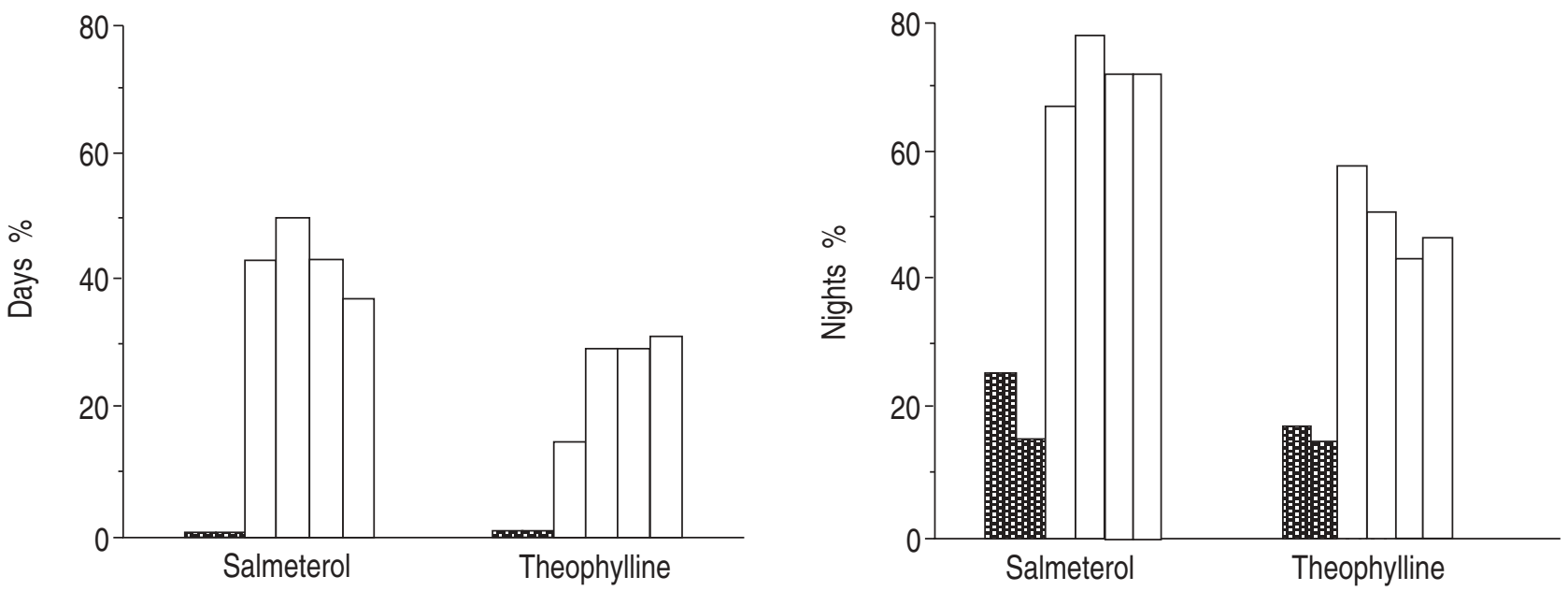

Fig. 1. - Median of the percentage of days and nights with symptom score equal to 0 during two run-in weeks and four active treatment weeks, in subjects treated with salmeterol $(50 \mu \mathrm{g}$ b.i.d.) or with an individually titrated dose of oral theophylline. The decrease in nocturnal symptoms from the second run-in week to active treatment weeks was significantly greater for salmeterol than for theophylline. 䀠沮明: run-in; $\square$ : treatment.

but the improvement in symptoms was higher for patients treated with salmeterol than for patients treated with theophylline. In particular, the percentage of days and nights with asthma score $=0$ markedly increased in the salmeterol group (fig. 1) from $14 \%$ during the second run-in week to $71 \%$ during the 4 th week of active treatment. In subjects treated with theophylline, the percentage of days and nights with asthma score $=0$ also increased during active treatment, but to a lesser degree, from 14 to $46 \%(\mathrm{p}=0.044)$.

Additional daytime and night-time salbutamol medication was evaluated as a marker of the effect of the two treatments. Before randomization, use of rescue salbutamol was present at least once a day in all subjects during the day and in less than $50 \%$ of subjects during the night (fig. 2). During treatment with salmeterol, the median of the percentage of days without additional salbutamol significantly increased from 0 to $43 \%$ in the 3 rd week, and the median of the percentage of nights without additional salbutamol increased from 36 to $86 \%$ in

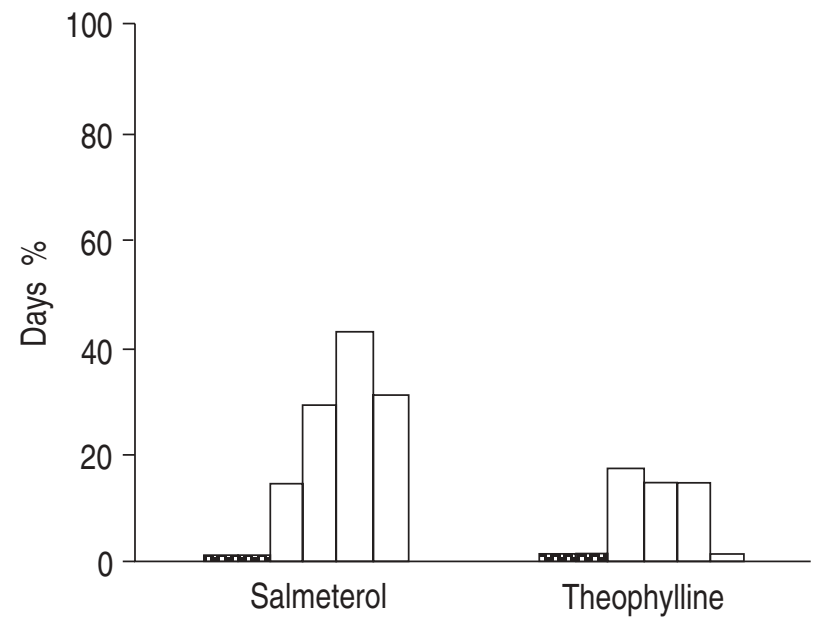

the 2nd run-in week. A significant increase in the same index was also observed in subjects treated with theophylline; comparison between the two treatments demonstrated a significant benefit following salmeterol treatment $(\mathrm{p}=0.002)$.

Morning and evening PEF measurements confirmed the efficacy of both treatments (fig. 3 ). In the salmeterol group, mean morning PEF increased from $337 \mathrm{~L} \cdot \mathrm{min}^{-1}$ during the 2 nd run-in week to $372 \mathrm{~L} \cdot \mathrm{min}^{-1}$ during the final 3 weeks of treatment, and evening PEF increased from 380 to $398 \mathrm{~L} \cdot \mathrm{min}^{-1}$, respectively. In the theophylline group, the increase was from 332 to $357 \mathrm{~L} \cdot \mathrm{min}^{-1}$ for morning PEF, and from 373 to $384 \mathrm{~L} \cdot \mathrm{min}^{-1}$ for evening PEF. The mean difference between treatments (salmeterol - theophylline), after adjustment for the difference in baseline value and country, was $10 \mathrm{~L} \cdot \mathrm{min}^{-1}$ for morning PEF, and $6 \mathrm{~L} \cdot \mathrm{min}^{-1}$ for evening PEF; this difference, however, was not significant $(\mathrm{p}=0.137$ for morning PEF; $\mathrm{p}=0.389$ for evening PEF). Diurnal variation (morning $\mathrm{PEF}$ - evening PEF of the following day) decreased from

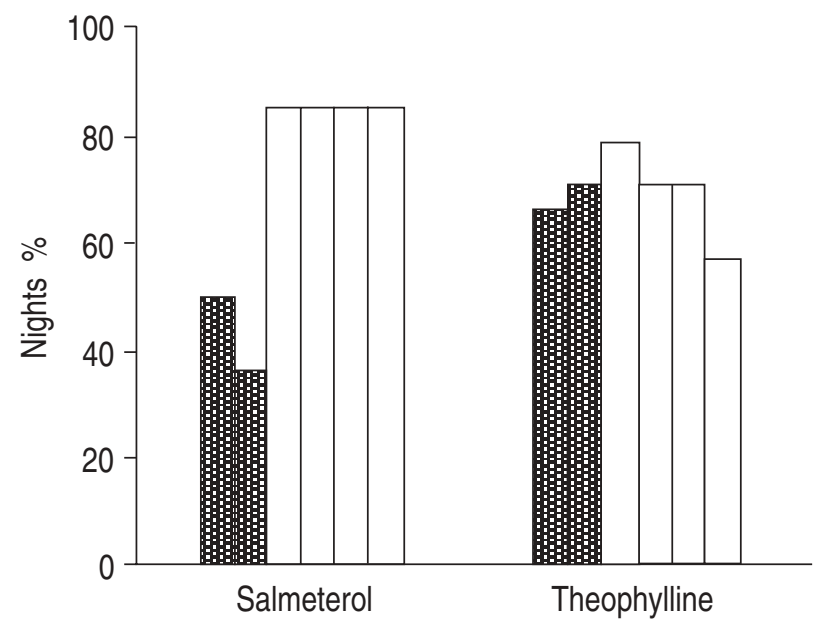

Fig. 2. - Median of the percentage of days and nights without additional use of rescue salbutamol during two run-in weeks and four active treatment weeks, in subjects treated with salmeterol $(50 \mu \mathrm{g}$ b.i.d.) or with an individually titrated dose of oral theophylline. The increase in the percentage of nights with no additional medication from the second run-in week to active treatment weeks was significantly greater for salmeterol than for theophylline. 

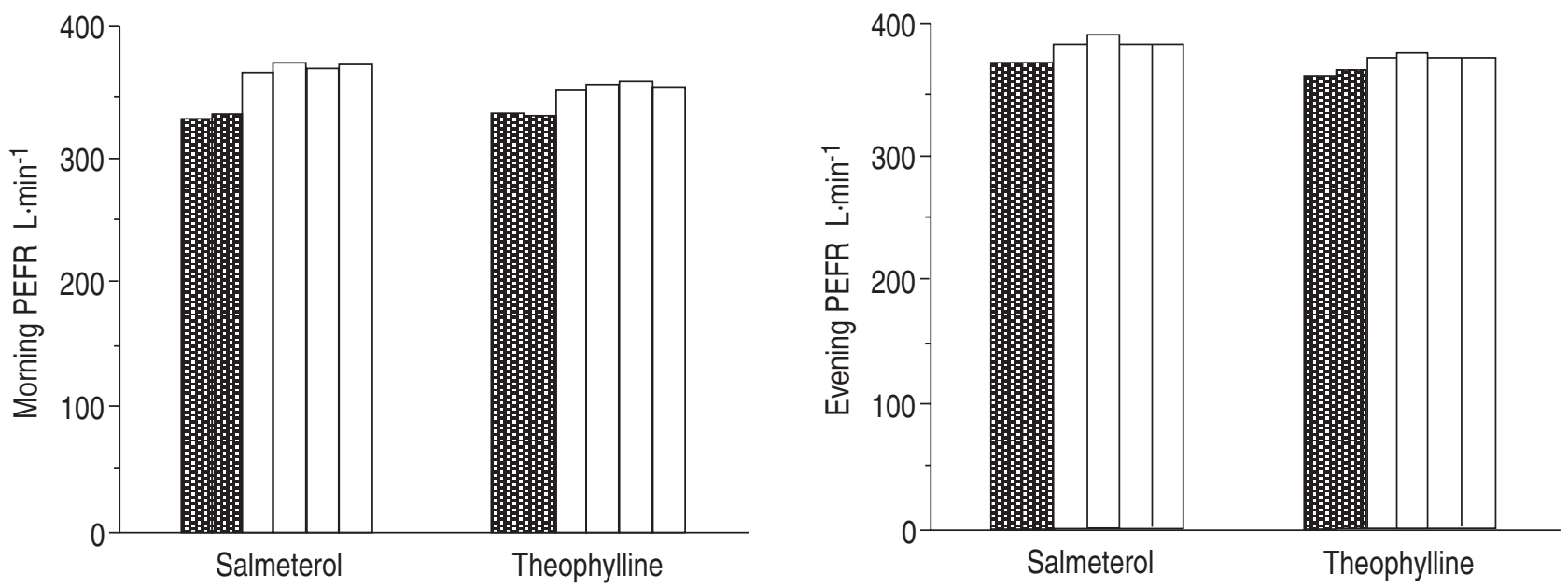

Fig. 3. - Mean values for morning and evening peak expiratory flow rate (PEFR) during two run-in weeks and four active treatment weeks, in

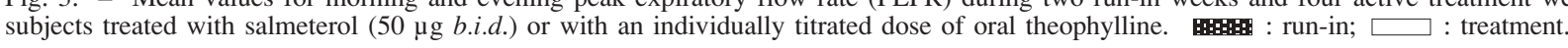

the 2 nd run-in week to the last 3 weeks of treatment in both groups; patients receiving salmeterol showing slightly less diurnal variation (mean adjusted $20 \mathrm{~L} \cdot \mathrm{min}^{-1}$ ) than theophylline-treated patients $\left(25 \mathrm{~L} \cdot \mathrm{min}^{-1}\right)$, but again this difference was not statistically significant $(\mathrm{p}=0.387)$.

The distribution of serum levels of theophylline measured in the peripheral venous blood, obtained by puncture, at the beginning of the active treatment and at the end of active treatment are reported in figure 4. More than $60 \%$ of subjects in both groups reached a serum theophylline level in the therapeutic range $\left(10-20 \mu \mathrm{g} \cdot \mathrm{mL}^{-1}\right)$ at the end of the titration period and at the beginning of active treatment; only five subjects showed serum theophylline level $<5 \mu \mathrm{g} \cdot \mathrm{mL}^{-1}$. At the end of active treatment, the serum theophylline level was close to zero in the salmeterol group, while the percentage of subjects with a therapeutic concentration of theophylline was reduced to $43 \%$ in the theophylline group. However, only nine subjects showed serum theophylline level $<5 \mu \mathrm{g} \cdot \mathrm{mL}^{-1}$. The mean value of serum theophylline concentration in the 97 subjects treated with theophylline was slightly but significantly reduced from the beginning to the end of the treatment period $\left(10.9 \pm 4.2\right.$ vs $9.0 \pm 4.6 \mu \mathrm{g} \cdot \mathrm{mL}^{-1}$ after 2 weeks vs $9.8 \pm 4.9 \mu \mathrm{g} \cdot \mathrm{mL}^{-1}$ after 4 weeks; $\mathrm{p}=0.02$ ).

FEV1 and FVC slightly increased during both treatments. FEV1 increased from 2.26 to $2.51 \mathrm{~L}$ following salmeterol treatment, and from 2.27 to $2.39 \mathrm{~L}$ following theophylline treatment. There was no significant difference between treatment groups at any time during active treatment.

\section{Withdrawals and adverse events}

Nine patients were withdrawn from the study after randomization because of adverse events or asthma exacerbation: four patients were randomized to salmeterol and five to theophylline. No serious adverse event was reported in relation to the study drugs.

The rate of adverse events during active treatment was similar in both groups, with a slightly greater prevalence in theophylline-treated patients $(28$ patients in the theophylline group vs 21 patients in the salmeterol group; 36 events vs 26 events). Increase in asthma symptoms
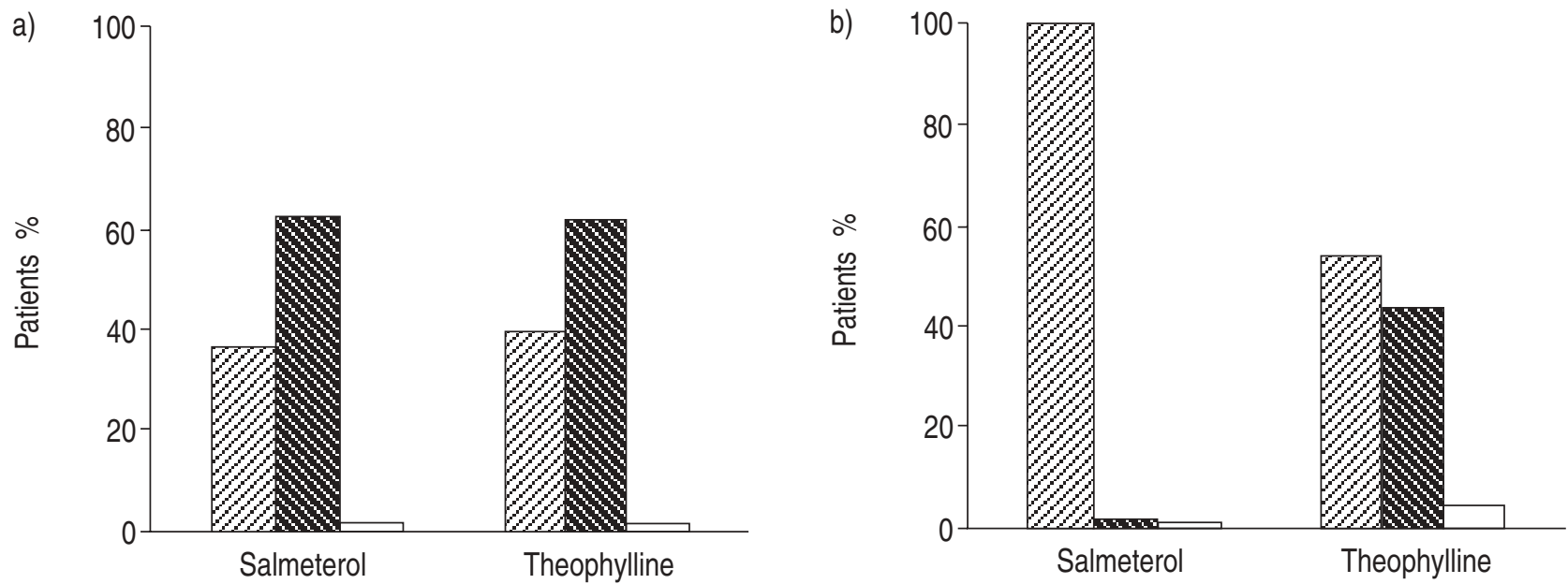

Fig. 4. - Percentage distribution of classes of serum levels of theophylline (obtained on frozen serum samples by the central laboratory) in sal-

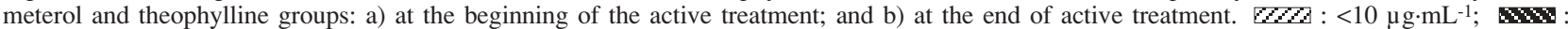
$10-20 \mu \mathrm{g} \cdot \mathrm{mL}^{-1} ; \quad \square:>20 \mu \mathrm{g} \cdot \mathrm{mL}^{-1}$. 
Table 3. - Incidence of adverse events during active treatment with salmeterol or theophylline

\begin{tabular}{lcccc}
\hline & \multicolumn{2}{c}{$\begin{array}{c}\text { Salmeterol } \\
(\mathrm{n}=92)\end{array}$} & \multicolumn{2}{c}{$\begin{array}{c}\text { Theophylline } \\
(\mathrm{n}=97)\end{array}$} \\
& Patients & Events & Patients & Events \\
\hline Tremor & 0 & 0 & 0 & 0 \\
Headache & $1(1 \%)$ & 1 & $2(2 \%)$ & 2 \\
Palpitations & $1(1 \%)$ & 1 & 0 & 0 \\
Muscle cramps & 0 & 0 & 0 & 0 \\
Tachycardia & $1(1 \%)$ & 1 & 0 & 0 \\
Gastric irritation & 0 & 0 & $7(7 \%)$ & 7 \\
Nausea and vomiting & 0 & 0 & $2(2 \%)$ & 2 \\
Diarrhoea & 0 & 0 & 0 & 0 \\
Insomnia & 0 & 0 & 0 & 0 \\
\hline
\end{tabular}

was reported during active treatment by seven patients treated with salmeterol, and by six patients treated with theophylline. The incidence of predictable adverse events due to the study drugs during treatment was slightly greater in the theophylline group $(11 \%)$ than in the salmeterol group (3\%), mainly because of the presence of symptoms of gastric irritation (table 3).

No change in blood pressure and pulse rate was observed in either treatment.

\section{Discussion}

This multicentre study shows that inhaled salmeterol, at the usual recommended doses, was at least as effective as slow-release oral theophylline individually titrated to maintain a serum theophylline level of $10-20 \mu \mathrm{g} \cdot \mathrm{mL}^{-1}$, in controlling symptoms and in improving PEF in adult patients with moderate-to-severe asthma. Furthermore, salmeterol had a better effect than theophylline on nocturnal symptoms, with most nights during active treatment being without symptoms or rescue salbutamol. Salmeterol also appeared to be better tolerated than theophylline at these dosages.

During recent years, several guidelines for asthma treatment have been published [7-9]; although all of them agree that inhaled anti-inflammatory drugs must be recommended as the first-line treatment of moderate-to-severe asthma, regular bronchodilator drug use is suggested when asthma symptoms and functional abnormalities are not completely controlled by regular use of cromoglycate or inhaled steroids [7-9]. In these cases, the choice is usually between slow-release oral theophylline and inhaled or oral $\beta_{2}$-agonists. In the last case, a persistent bronchodilation can be obtained by traditional short-acting $\beta_{2^{-}}$ agonists, administered four times daily by inhalation or two times daily by oral route. Oral controlled-release preparations of $\beta_{2}$-agonist have been proven to be as effective as oral theophylline but they frequently cause side-effects, such as tremor and restlessness $[10,11]$. On the other hand, short-acting inhaled $\beta_{2}$-agonists have frequently been shown to be less effective than theophylline, because of their short duration and low compliance of patients [12].

Long-acting inhaled bronchodilators, like salmeterol, have been proven to allow a persistent bronchodilation for at least $12 \mathrm{~h}$, and could easily be used b.i.d. by the patient in the long-term treatment of asthma. The benefit is persistent after 12 months of regular therapy [13]. Therefore, one of the many issues to address when a regular bronchodilator drug must be used in the long-term treatment of asthma, is the choice between oral slow-release theophylline and a long-lasting inhaled $\beta_{2}$-agonist. From a therapeutic point of view, inhaled therapy is preferred to the oral route because of the higher local potency and the lower level of systemic side-effects, but other problems, such as the relative efficacy of the two treatments, patient compliance, and possible side-effects, are also relevant to this choice. Furthermore, the cost of the treatment with two different drugs should be considered, oral theophylline showing the lower cost.

This study shows that salmeterol has a better effect on nocturnal symptoms and use of rescue salbutamol drug during the night than theophylline, and is at least as effective as theophylline in controlling daytime symptoms. Furthermore, the effects of both drugs on pulmonary function are equivalent. The changes in PEF seen during active treatment with salmeterol and theophylline, in this study, were relatively small (i.e. mean change in morning PEF 36 and $25 \mathrm{~L} \cdot \mathrm{min}^{-1}$, respectively) and might not be considered to be of clinical importance. This fact may also explain the lack of a significant difference in efficacy between the two treatments, and it could be explained by the stability of the disease at the start of the study medications. In effect, more than $60 \%$ of the patients were under regular treatment with inhaled or oral anti-inflammatory drugs during the run-in period, and, consequently, no large increase in PEF could be expected in these patients during treatment.

A level of serum theophylline in the therapeutic range was obtained in two thirds of the patients at the beginning of active treatment, and in half of patients at the end of 4 weeks of treatment. This fact can be partially explained by a tendency of the method used for the titration of serum theophylline (Acculevel) to overestimate the serum concentration of theophylline with respect to the measurements obtained on frozen serum sample by the central laboratory, and partially by a low compliance of the patients in taking oral slow-release theophylline regularly and for a long time. The difficulty in obtaining a serum theophylline level $>10 \mu \mathrm{g} \cdot \mathrm{mL}^{-1}$ in the long-term treatment of reversible airway obstruction is well-known, because of the difficulties in using this drug [14]. However, the therapeutic benefit of theophylline in treating airways obstruction is dose-related, and can be observed at levels as low as $5 \mu \mathrm{g} \cdot \mathrm{mL}^{-1}$, particularly when theophylline is given in conjunction with $\beta_{2}$-agonists $[15$, $16]$. In our study, only nine subjects in the theophylline group showed a serum theophylline concentration $<5$ $\mu \mathrm{g} \cdot \mathrm{mL}^{-1}$ at the end of the active treatment, suggesting that compliance with the treatment was acceptable.

The low compliance of patients to taking oral theophylline regularly could be due, in part, to the well-known side-effects of this drug, which frequently limit the adequate intake of the drug. In our study, 27 adverse events due to theophylline (headache, gastric irritation, nausea and vomiting, diarrhoea and insomnia) were reported in 243 patients during the titration period, and 11 events in 97 patients during the active treatment with theophylline. This percentage could have been higher since almost half of the patients had previously been treated with oral theophylline, and were probably already tolerant to the drug. 
However, we cannot exclude that a similar low compliance could also have been present in the salmeterol group at the end of active treatment. This fact could further suggest that salmeterol had a better efficacy compared to theophylline.

Although the inclusion criteria of the study required a positive response to bronchodilator and a baseline FEV1 greater than $50 \%$ of the predicted value, a small number of subjects did not satisfy these inclusion criteria. When the bronchodilator response was assessed taking into account the baseline level (e.g. as change in FEV1\% predicted), the number of subjects not responsive to bronchodilator was still low. Therefore, we think that the inclusion of these subjects with partially reversible chronic obstructive pulmonary disease (COPD) should not have any relevant influence on the results obtained.

In conclusion, inhaled salmeterol seems to be more effective and better tolerated than oral theophylline in the long-term treatment of reversible airway obstruction. Inhaled salmeterol should be considered as a first choice when a bronchodilator drug must be added to the regular use of anti-inflammatory therapy in a patient with uncontrolled asthma.

Principal investigators participating in this study: Eire: B. Keogh, L. Clancy (Dublin); The Netherlands: A.R.J. van Keimpema (Amsterdam), H.C.H. Gooszen (Eindhoven), W. van den Berg (Nijmegen); Italy: R. Dal Negro (Bussolengo), P.L. Paggiaro (Pisa), C.F. Donner (Veruno), E. Gramiccioni (Bari); Norway: A. Bergmann (Oslo), A. Gulsvik (Bergen), H. Ringdal (Molde); Spain: J. Duce (Zaragoza), M. Martinez (Valencia); West Germany: M. Peters (Hamburg), J. Napp (Lübeck), W. Weede (München), J. Stauder (Mainz), K. Franke (Schloss Holte-Stekenbrock), H. Trauth (Marburg), K. Bonicke (Lienen), D.H. Mahlo (Berlin); Portugal: D. Borges (Funchal), A. Ramalho de Almeida (Vila Nova de Gaia).

\section{References}

1. Brogden RN, Faulds D. Salmeterol xinafoate: a review of its pharmacological properties and therapeutic potential in reversible obstructive airway disease. Drugs 1991; 42: 895-912.

2. Paggiaro PL, Giannini D, Bacci E, Vagaggini B. Clinical effects of salmeterol, a new long-acting $\beta_{2}$-agonist. Eur Respir Rev 1993; 3(14): 391-395.
3. Weinberger M. Pharmacology and therapeutic use of theophylline. J Allergy Clin Immunol 1984; 73: 525-540.

4. Fjellbirkerland L, Gulsvik A, Palmer JBD. The efficacy and tolerability of inhaled salmeterol and individually dose-titrated, sustained release theophylline in patients with reversible airway disease. Respir Med 1994; 88: 599-607.

5. Muir JF, Bertin L, Georges D. Salmeterol versus slowrelease theophylline combined with ketotifen in nocturnal asthma: a multicentre trial. Eur Respir J 1992; 5: 1197-1200.

6. Quanjer PhH, Yammelin GJ, Cotes JE, Pedersen OF, Peslin R, Yernault J-C. Lung volumes and forced ventilatory flows. Report Working Party "Standardization of Lung Function Tests", European Coal and Steel Community. Eur Respir J 1993; 6 (Suppl. 16): 5-40.

7. NHLBI. International consensus report on diagnosis and treatment of asthma. Eur Respir J 1992; 5: 601-641.

8. NHBLI. Guidelines for the diagnosis and management of asthma. J Allergy Clin Immunol 1991; 88: 425-533.

9. Hargreave FE, Dolovich J, Newhouse MT. The assessment and treatment of asthma: a conference report. $J$ Allergy Clin Immunol 1990; 85: 1098-1111.

10. Laursen LC, Taudorf E, Grosspelius Y, Gymose E, Weeke B. Long-term oral therapy of asthma with terbutaline and theophylline, alone and combined. Eur J Respir Dis 1985; 66: 82-90.

11. Pierson WE, La Force CF, Bell TD, MacCosbe PE, Sykes RS, Tinkelman D. Long-term, double-blind comparison of controlled-release albuterol versus sustained-release theophylline in adolescents and adults with asthma. $J$ Allergy Clin Immunol 1990; 85: 618-626.

12. Joad JP, Ahrens RC, Lindgren SD, Weinberger MM. Relative efficacy of maintenance therapy with theophylline, inhaled albuterol, and the combination for chronic asthma. J Allergy Clin Immunol 1987; 79: 78-85.

13. Britton MG, Earnshaw JS, Palmer JBD. A twelve month comparison of salmeterol with salbutamol in asthmatic patients. Eur Respir J 1992; 5: 1062-1067.

14. Addis GJ, Johnston IDA. Theophylline in the management of airway obstruction. BMJ 1990; 300: 928-931.

15. Whiting B, Kelman AW, Struthers AD. Prediction of response to theophylline in chronic bronchitis. $\mathrm{Br} \mathrm{J} \mathrm{Clin}$ Pharmacol 1984; 17: 1-8.

16. Fairshter RD, Busse WW. Theophylline: how much is enough? J Allergy Clin Immunol 1986; 77: 646-648. 